\title{
O ÚLTIMO LENIN E AS BATALHAS CONTEMPORÂNEAS DA AMÉRICA LATINA
}

\author{
EL ÚLTIMO LENIN Y LAS BATALLAS CONTEMPORÁNEAS EN AMÉRICA LATINA
}

\author{
THE LAST LENIN AND CONTEMPORARY BATTLES IN LATIN AMERICA
}

DOI: http://dx.doi.org/10.9771/gmed.v12i2.39013

Jaime Ortega ${ }^{1}$

Resumo: O modo como Lênin tem sido relacionado a todo o marxismo é o que chamamos de "junção teórica". Este texto apresenta dois momentos em que essas conjunturas surgiram em torno do trabalho de Lenine e sugere uma possível alternativa às condições e problemas que a esquerda latino-americana teve. Foca, no final, a noção do último Lênin, para se referir aos problemas da transformação do Estado em sua relação com a economia, bem como à especificidade de sua construção.

Palavras-chave: Lenin. Política. Estado. Economia. América Latina.

Resumen: La forma en que se ha relacionado a Lenin con el conjunto del marxismo es lo que denominamos una "coyuntura teórica". Este texto presenta dos momentos en los que se han dado estas coyunturas en torno a la obra de Lenin y sugiere una posible alternativa frente a las condiciones y problemas que ha tenido la izquierda latinoamericana. Se centra, hacia el final, en la noción del último Lenin, para referir a los problemas de la transformación del Estado en su relación con la economía, así como la especificidad de la construcción de este.

Palabras clave: Lenin. Política. Estado. Economía. América Latina.

Abstract. The way in which Lenin has been related to the whole of Marxism is what we call a "theoretical juncture". This text presents two moments in which these conjunctures have arisen around Lenin's work and suggests a possible alternative to the conditions and problems that the Latin American left has had. It focuses, towards the end, on the notion of the last Lenin, to refer to the problems of the transformation of the State in its relationship with the economy, as well as the specificity of its construction.

Keywords: Lenin. Politic. State. Economy. Latin America.

La referencia puntual que refiere al último segmento de la obra de Karl Marx ha sido utilizada en numerosas y diversas ocasiones. Theodor Shanin refirió al "Marx tardío" (1990) para comprender "la vía rusa"; Enrique Dussel bautizó un conjunto amplio de documentos a partir de la noción de "el último Marx" (1990); con el mismo nombre, recientemente, Macello Musto (2020) relató el trayecto del último viaje del Moro, cuyo destino fue Argelia. La figura de "lo último" es muy útil, pues ha permitido reflexionar sobre momentos minoritarios dentro del corpus textual, en este caso de Marx.

En el caso que aquí nos convoca queremos pensar en y con el último Lenin, con lo que hacemos referencia a la producción teórica del revolucionario ruso en su calidad de constructor del Estado socialista y ya no sólo como opositor al zarismo u organizador del partido bolchevique. Ello aleja tanto su 
evaluación del capitalismo del siglo XIX expresado en su Desarrollo del capitalismo en Rusia, así como los fragmentos más conocidos de extensa obra, como el ¿Qué hacer?

Se trata de un segmento menos conocido y que se encuentra atravesado por diversas discusiones, que, a pesar de sus especificidades, apuntalan a la movilización de un conjunto de temáticas relevantes para la izquierda contemporánea. El silenciamiento que sufrió Lenin por parte de las izquierdas, quienes cedieron ante la presión del liberalismo, nos impidió acceder a un conjunto de planteamientos, que, leídos hoy en América Latina, resultando por demás cruciales. Así, 150 años después de su nacimiento, celebramos a Lenin recurriendo a su producción última, aquella que permite pensar su tiempo, pero también el nuestro.

El tratamiento dado por el liberalismo a Lenin como el precursor de un sistema totalitario, que ahogaba la libertad individual, fue en gran medida aceptado por la izquierda en América Latina, que lentamente abandonó la referencia teórica del ruso. Esto contribuyó a colocar a Antonio Gramsci como eje central de una práctica política así como el referente más socorrido en los peores años del vendaval neoliberal. Ciertamente, Gramsci se presentaba para la coyuntura abierta con los procesos de democratización y cambio del patrón de acumulación en la década de 1980, como el teórico más adecuado. Ante el diluvio neoliberal, que arrasó a las viejas izquierdas -incluido el poder estatal soviéticoel refugio gramsciano permitió resistir y navegar en contra de la marea. Sin embargo, ante las nuevas quiebras del liberalismo (en su versión "neo"), vuelve la inquietud y la necesidad de recurrir al corpus textual de Lenin, como orientación y brújula y no como verdad última.

El siglo XXI inició en América Latina con el reforzamiento de nuevos planteamientos emancipatorios, los cuales tuvieron que responder pragmáticamente a la ofensiva del mercado mundial, empeñada en mantener el neoliberalismo. Los múltiples retos que las experiencias "progresistas" o de la "marea rosa" (término popularizado en la academia anglosajona) dejaron en la región, nos convocan a preguntarnos por el sentido que guarda, en el campo teórico, la producción marxista. ¿Servía el marxismo para algo más que para alimentar pequeñas sectas políticas? ¿Puede el marxismo decir algo más, además de su pertinente denuncia de las formas de explotación? ¿Contribuye en algún punto de su largo recorrido a la construcción social de un orden alternativo y no sólo de la resistencia? Finalmente, ¿Puede aun el marxismo decir algo de estos imbricados procesos en donde la "bella contradicción entre capital y trabajo" (ALTHUSSER, 1974, p.85) no aparece sino a condición de los elementos de sobredeterminación? ¿Es posible acompañar las motivaciones de la lucha por la hegemonía -aprendidas con Gramsci- con otro nivel de construcción de lo político-estatal?

Sostenemos que Lenin permite acceder a un conjunto de registros que clarifican la especificidad del espacio de la teoría política, de la disputa y construcción del poder. Ello implica, por supuesto, un ejercicio de lectura, una operación teórica, pues se trabaja sobre condiciones sociales y políticas más diversificadas a las que él enfrentó hace un siglo. Pero la distancia temporal no invalida el campo del pensamiento, menos aun cuando se hace notar que en el terreno de la lógica política hay persistencias significativas. Las lógicas sociales modernas, que verifican cambios de fisonomía, también anclan 
elementos significativos persistentemente. Lenin sirve, porque la persistencia de dichas formas de operación de la sociedad capitalista.

El texto se expone a partir de tres segmentos. El primero aborda el nacimiento del "leninismo", es decir, refiere a una primera coyuntura teórica de lectura, en donde la disputa por el poder afianza una forma ideológica a partir de determinadas señas de identidad, se trata de una operación teórica que alejará a Lenin de la obra de Marx. El segundo apuntala la coyuntura teórica que fue producida en América Latina al calor de la "actualidad de la revolución", es decir, aquella marcada por el impacto de la revolución cubana y que termina con la emergencia de los procesos de democratización. Finalmente, proponemos una forma de abordar a Lenin, permitiendo pensar a partir de a coyuntura contemporánea.

Lenin no está de moda, tampoco tiene buena fama, no es un momento productivo extendido, ni siquiera entre los marxistas. La losa del "leninismo", es decir, de la osificación de un poder que devino ideología, pesa demasiado a algunos. A ello debe entenderse, además, las transformaciones en el seno de la sociedad capitalista. Lenin aparece más con un teórico de la época inicial del fordismo; sin embargo, esto puede ser cuestionado. Lo que pretendemos aquí es sacar a Lenin del encierro temporal y trabajar teóricamente con su obra. Es cierto que existen registros de su obra que hoy se encuentran superados, por ejemplo, la excesiva confianza en la neutralidad de la ciencia y la técnica que se desprende de su deseo por la electrificación; sin embargo, ello no obsta que en condiciones de colonialidad, la ciencia y la técnica puedan tener alguna utilidad en la conformación de espacios de soberanía de los pueblos nacionales o multi nacionales, como lo demostró recientemente Doménico Losurdo (2019).

Debe aclararse que esta reflexión no pretende plantear que hay un "exceso" del giro hacia Gramsci que se dio en la producción marxista latinoamericana, que se encuentra vinculada a la etapa de resistencia al neoliberalismo. El teórico italiano era imprescindible para pensar la coyuntura latinoamericana de oposición al periodo neoliberal y resultó importante en la época donde el reflujo imperio o bien donde se dio un lento pasó a la ofensiva de las izquierdas. Sin embargo, el relativo olvido de Lenin, su eclipsamiento como teórico - aunque se le reconoció como un político u organizador- han restado densidad a una reflexión que no se encuentra ni en Gramsci, ni en Rosa Luxemburgo, ni en otros teóricos: la ardua tarea de la construcción de poderes sociales asociados a los procesos de emancipación. Esta sola razón, debería llamar la atención en medio de la búsqueda incesante de brújulas para pensar no solo los momentos de oposición, sino también los de construcción social.

\section{El nacimiento del leninismo: la primera coyuntura teórica}

Estableceremos bajo el nombre de "coyunturas teóricas" las formas diversificadas de lectura y trabajo teórico con respecto a un autor o conjunto de autores, que dan respuesta o permiten plantear preguntas ante determinadas condiciones sociales. Retomamos de Althusser (1974, p.IX) este concepto, que alude directamente a las condiciones de posibilidad, tanto al seno de la lucha política como de los avances de la teoría marxista. Es decir, en algunos momentos o periodos, un conjunto de esfuerzos de producción giran en torno a la misma problemática e incluso lo hacen con el mismo lenguaje. Ella implica 
un conjunto de coordinas dispuestas a partir de las cuales se lee a un autor, se le tensiona y se produce entorno a él. No es posible considerar siempre la producción teórica de manera indistinta, existen diversos acontecimientos que establecen los límites o posibilidades de ella.

Una primera coyuntura teórica es la que se ubica en el periodo posterior a la revolución rusa (1917-1928) y la muerte del propio Lenin. Las condiciones de producción y lectura en este periodo dan como resultado el concepto de "leninismo". Esta etiqueta, que marcará la historia del movimiento comunista y de gran parte del marxismo, tiene su origen en la lucha por el poder en la Unión Soviética. Ello es perceptible a partir de numerosas producciones, que en esta época marcaron la línea por a través de la cual se comprendería la obra de Lenin en adelante. De tal forma, que la primera sea distintiva es que se trata de una construcción alrededor de la lucha por el poder en el naciente Estado soviético.

Cuatro fueron los autores que contribuyeron a establecer esta seña de identidad, signada por la estabilización del Estado soviético, después de una cruenta guerra civil -que incluyó, además, invasiones extranjeras- y de proceso de desarticulación de la economía, prácticamente en su totalidad. El más famoso de estos trabajos fue el de José Stalin, el entonces dirigente georgiano, pieza clave de la construcción del Estado y después su máximo conductor. José Stalin, se sabe, popularizó la acepción "leninismo" a partir de su conferencia titulada "los fundamentos del leninismo". Si bien versiones críticas han responsabilizado al georgiano por el invento teórico que este significó al imbricarlo a las disposiciones del aparato estatal, no fue el único responsable.

Podemos ubicar producciones que corren coetáneamente. Es el caso de la conferencia de Nicolas Bujarin, uno de los teóricos del Partido bolchevique, quién en su Lenin marxista, delinea la especificidad de la producción teórica del dirigente revolucionario: la cualidad de producirse en una "época diferente" (BUJARIN, 1978, p.21) a la de Marx y Engels, con el predominio del capital financiero, el anuncio del derrumbe del capitalismo y el ascenso del dominio de la clase obrera. Bujarin, además, colocó atención en el problema del Estado y de la alianza con el mundo campesino. Se trató, sin duda, de uno de los textos más cuidados, pero como veremos adelante, no alejado de una órbita de reflexión.

Además de Bujarin, Giorgi Zinoviev participó en la construcción del leninismo. Tanto en la conferencia titulada Lenin -dictada cuando este sufrió un atentado por parte de una anarquista- como en el texto El leninismo. La primera es una biografía del dirigente ruso, narrada de manera lineal. El segundo trabajo si expresa ya una concepción más global, que parte el cuestionamiento del uso del epíteto de "leninismo" por el propio Lenin, pero que, al final, Zinoviev termina aceptando. Además de definir a marxismo como una teoría totalizante que "abarca todos los problemas de la vida y de la evolución humana" (ZINOVIEV, 1977, p.144), cuestión ya problemática y cuestionable por sus implicaciones, asume una definición que vale la pena señalar:

El leninismo es, ante todo, la comprensión, la explicación marxista, de los nuevos estudios históricos de la evolución de la sociedad de la nueva experiencia del movimiento obrero mundial (y del movimiento revolucionario en general), de todo lo que surgió después de Marx y en parte ha sido deformado por los teóricos oficiales de la II Internacional. El leninismo es la teoría y la práctica marxista en la época del imperialismo (es decir del capitalismo monopolista en vías de deterioro) y de la revolución en ascenso" (ZINOVIEV, 1977, p.144). 
Zinoviev no pasó a la historia como un gran teórico y mucho menos como un político habilidoso, fue defenestrado junto a Bujarin. Un caso especial es el de Trotsky, el único de los aquí aludidos a partir del cual se creo una corriente que se expandió de manera diversificada, creando a su paso numerosos intentos de reactualizar el "bolchevismo-leninista", como gustó de nombrar a su corriente. El viejo líder del Ejército Rojo encaró directamente la definición de Stalin en su texto "Las tendencias filosóficas del burocratismo" (2004). Más allá de su enfrentamiento -esperado a final de la década de 1920 - Trotsky nunca dejó de utilizar el "leninismo" como una realidad, es decir, contribuyó a su establecimiento. Lo hizo de dos formas, tanto apuntalando su cercanía con el líder revolucionario y su pensamiento que era una "lámpara del leninismo" (TROTSKY, 1970, p.148) como formulando una versión de conjunto del marxismo como cosmogonía.

Enmarcado las discusiones alrededor de la coyuntura es posible definir mejor las condiciones de emergencia del "leninismo": "El leninismo es el marxismo de la época del imperialismo y de la revolución proletaria. O más exactamente: el leninismo es la teoría y la táctica de la revolución en proletaria en general, la teoría y la táctica de la dictadura del proletariado en particular" (STALIN, 1978, p.73). A partir de esta construcción es posible tejer los elementos que se jugaron en torno a esta coyuntura teórica y en la que, a pesar de las diferencias supuestas o reales, se tejió una forma de entender el aporte de Lenin.

La de Stalin fue la más clara osificación del pensamiento de Lenin en una ideología de Estado, misma que marcó la pauta, insistimos, aun cuando Trotsky le opusiera formalmente, su propio desarrollo mostró concordancia. Esto es así porque se colocaban elementos que trascendían a las individualidades. Las señas de identidad son las siguientes: primero, que la época en la que emerge el liderazgo y la teorización de Lenin es la del imperialismo. Segunda, que está marcada por el desarrollo de la clase obrera madura, con sus instrumentos políticos, principalmente el partido, ausentes previamente. Tercera, que este pensamiento era propio de la construcción de una forma de Estado. Teniendo estos elementos claves, es posible pensar una época entera de producción teórica alrededor de lo que comúnmente se denomina "marxismo-leninismo". Cuestión que generó múltiples consecuencias.

Señalemos ahora algunos de los efectos de la elección de estos elementos como las marcas de identificación del "leninismo". En primer lugar, que el imperialismo era una fase histórica nueva, dominada por el capital financiero y los monopolios, diferente a la que Marx, que, supuestamente, sólo pudo observar y retratar en El Capital, este último sería un texto que hablaría de la "época preimperialista" o de "la libre competencia". Así, en una lectura historicista, la obra de Marx quedaría atrapada en un conjunto de condiciones que habrían sido ya superadas por el concepto de imperialismo. No sorprende que ni Stalin, ni Trotsky, ni Zinoviev, produjeran algún tratado, comentario o uso significativo de El Capital.

El segundo elemento refiere a la distinción que se hace a partir de la "revolución proletaria". Stalin argumentó que la época de Marx y de Engels estuvo marcada por un desarrollo incipiente, aun identificado con el artesanado, antes que con la clase obrera industrial. Lenin intervino en una época en donde la clase trabajadora ya se habría desarrollado plenamente y se encontró en condiciones de tomar la Germinal: Marxismo e Educasãa em Debate, Salvador, v. 12, n. 2, p. 86-100, out. 2020. ISSN: 2175-5604 90 
dirección del Estado, a partir de la construcción de un instrumento ausente. De ahí que entre Marx y Lenin surgiera una diferencia: la teoría del partido. El surgimiento de este campo, tan explotado por Stalin y demás dirigentes soviéticos, sólo fue posible, para los constructores del "leninismo", por la madurez del proletariado. Al final, todos compartían el fetiche de la forma partido como el instrumento único de la política comunista.

El último tema es el que refiere a la construcción del Estado. Aunque es el registro menos desarrollado, se asume que el Estado es necesario para la construcción del socialismo, quedando suspendida cualquier referencia proto-anarquista, como aquella que se podría leer en El Estado y la revolución. Al final, más allá de la disputa en torno al "socialismo en un solo país", era claro que había que defender la creación soviética de todas las amenazas que el mundo capitalista lanzaba en su contra.

Como puede notarse esta coyuntura está marcada por la construcción del poder tras la revolución soviética. Aparece entonces el leninismo como un momento universal, de una época, que en cierto sentido completa al marxismo en tanto que brinda herramientas exclusivas, centradas en un tiempo. Es una lectura historicista: Marx es el personaje del siglo XIX, Lenin el del siglo XX. El teórico alemán realizó su trabajo en una época "previa" a la gran industrialización; en tanto que el ruso lo hizo cuando ya existían las condiciones para la revolución. Con la fundación del "leninismo", se abría un hiato profundo entre Marx y la tradición que fundó.

\section{Segunda coyuntura de lectura: Lenin en América Latina}

Hacia finales de la década de 1950 se abre una nueva coyuntura en la que Lenin tendrá una gran recepción. Varios factores históricos permiten acceder a la comprensión de ella. En primer lugar, la crisis entre 1953-1956 en el campo socialista viene a ratificar algo que ya estaba sucediendo en los hechos, aunque débilmente: la mayor pluralización de las izquierdas. Si en Europa Occidental la tradición comunista tuvo centralidad suficiente en décadas previas, ella comienza a erosionarse tanto por los grupos extra parlamentarios, como por versiones diversas dentro del propio comunismo, además del resurgir consejista en zonas donde la izquierda suele estar aislada del movimiento popular. Esto se profundiza en regiones como América Latina, en donde el comunismo como corriente fue persistente, pero convivió con el nacionalismo popular, que, en la mayor de las ocasiones, arrastró tras de sí a la mayoría de la sociedad, bajo la lógica de construcción popular, antes que clasista. Si bien es cierto que este nacionalismo revolucionario había entrado en crisis, lo cierto es que se trató de una fuerza persistente entre los sectores subalternos. La revolución cubana de 1959 impulsó una serie de tendencias que ya ocurrían en el continente y en cuyo seno se encontró esta pluralización de las izquierdas.

Así, no resulta casual que Regis Debray definiera al "castrismo" como un "leninismo apresurado" (DEBRAY, 1976, p.1969), creando, con ello, un nuevo registro a partir del cual sería posible leer al teórico ruso. Sin embargo, es preciso referirse a los múltiples encuentros que tuvo Lenin con América Latina. El nacionalismo popular y revolucionario de las décadas de 1920 y 1930 recibieron al ruso no como un teórico, sino como un líder anti imperialista. Esto es comprobable en figuras como Haya de la Torre, 
Fausto Reinaga, Lázaro Cárdenas, Rómulo Gallegos, por mencionar sólo a algunos, en quienes Lenin pasó a ser un líder anti imperial, un organizador y un pedagogo de la política de masas. Para ellos era un líder que guio a su nación por la senda de la ruptura con el colonialismo, un dirigente que organizó un partido disciplinado, un pedagogo que enseñó hacer la revolución. A partir de 1959 el nombre de Lenin tomaría un nuevo cariz: el del teórico marxista de la política.

Esta es la segunda gran coyuntura de lectura que nos interesa en este texto. Ella trama los impulsos que dio la revolución cubana al transformar las condiciones socio-políticas de la región. Aquí, Lenin ya no fue una referencia santificada, sino un motivo de producción. Cierto es que la ruptura con el viejo "leninismo" no siempre acontece, dándose casos de repetición de lugares comunes. Pero también se aprietan las tuercas y comienza a surgir una perspectiva más amplia, en donde Lenin ya no es sólo un cúmulo de citas que revelan verdades absolutas. La interrogación sobre su obra se vuelve un ejercicio productivo.

En el campo económico persistió la vieja tradición de pensar a Lenin como el "marxista de nuestro tiempo". Es el caso de la obra del economista mexicano Alonso Aguilar Monteverde (1978), en donde el capitalismo monopolista es el concepto central que ocupa el lugar central. Algo similar se da con la obra de José Consuegra (1972), colombiano, quien interrogó a Lenin desde la noción clásica de imperialismo. Un avance se da con la obra del venezolano Vladimir Acosta que en su La teoría del desarrollo capitalista en Lenin (1977) quien encara las discusiones sobre los esquemas de reproducción de Rosa Luxemburg. La ruptura, en este campo específico, ocurrió con la teoría de la dependencia, que utilizó algunos referentes de la teoría del imperialismo, pero que no por ello renuncia a pensar desde los conceptos de El Capital de Marx.

Otro momento de esta segunda coyuntura lo representó la revolución cubana en su veta anti colonial. A través de revistas como Pensamiento Crítico o de Casa de las Américas, la lectura de Lenin despejó el camino del antiguo anti imperialismo, fácilmente confundido con un acuerdo pacífico con las burguesías "nacionales". La perspectiva impulsada desde la isla, permitió recuperar la memoria de las luchas anti coloniales con la veta presente en Lenin de los pueblos coloniales. Roberto Fernández Retamar, Carlos Rafael Rodríguez, Fernando Martínez Heredia, Thalía Fung, entre otros, acercaron a Lenin a dialogar con Martí, Fanon y Ho-Chi-Mihn.

Finalmente, a partir de múltiples puntos de encuentro, se llegó a conformar un corpus muy sugerente en donde Lenin fue leído como un teórico de la coyuntura, es decir, como aquel que aportó las categorías necesarias para el análisis político y los procesos de subjetivación. Una especie de ciencia de la coyuntura se desprendió a partir de determinadas obras que se produjeron a lo largo de la región, con resultados diversos: Nuñez Tenorio en su Lenin y la revolución (1968), Rodney Arismendi en su Lenin y la revolución en América Latina (1976), Carlos Cerda en El leninismo y la victoria popular (1972), Rudolf Sontag en su Marx y Lenin acerca de la sociología de la revolución (1974), Tomas Moulian en sus Cuestiones de teoría politica marxista: crítica de Lenin (2018), Álvaro García Linera en su Condiciones de la revolución social en Bolivia (De obreros, aymaras y Lenin) (1988), así como por Marta Harnecker en su La revolución social: Lenin y América Latina (1985). 
En este segundo momento de lectura se destacaron operaciones teóricas significativas. Si bien Lenin no deja de ser un gran revolucionario y ejemplo para generaciones de militantes de la revolución latinoamericana, lo cierto es que se dio paso a un tratamiento más sistemático de su obra. Las tensiones que provocó la necesidad de un instrumental teórico más refinado, así como las urgencias de la práctica política, convocaron a movilizar al dirigente ruso. Así, Lenin paseó por La Habana, por Buenos Aires, la ciudad de México, pero también por los campos, montañas y valles de América Latina, acompañando a los partidos, guerrillas y sindicatos de la región.

Dos procesos independientes, pero coincidentes en el espectro temporal terminaron con el pacto que se había forjado con Lenin. En el plano, el advenimiento de las discusiones de la "crisis del marxismo", que convocaban a pensar registros como la caducidad de figuras políticas como la "dictadura del proletariado", así como el considerar cierta inocencia de la técnica, como motivo fundamental del desarrollo de las fuerzas productivas, también, por supuesto, se dio un cuestionamiento más directo de la Unión Soviética y su régimen político y social. En el campo no teórico, América Latina vio derrotada cada una de sus intentonas revolucionarias y, salvo Nicaragua, en todos los países se dio la necesidad de una retirada de las perspectivas radicales. La democracia se impuso como el horizonte de sentido y la "actualidad de la revolución" perdió piso, tal como se le había conocido. Lenin fue sustituido por Gramsci. La aparición del teórico italiano en la caja de herramientas de las izquierdas renovó muchos de los sentidos y señas de identidad de esta, permitiendo resistir un poco mejor que sus pares europeos, el vendaval neoliberal y la crisis del socialismo. El periodo siguiente fue el de la recomposición y resistencia que asumió tanto la forma de una política autonomista -con el zapatismo a la cabeza- y la reestructuración electoral -a partir de la victoria de Hugo Chávez en 1999- alternadas con grandes movilizaciones en la región. Todo ello nos permite plantear entonces la pertinencia de la lectura del último Lenin, su pertinencia teórica en medio de fenómenos de construcción de poder. Todo esto debe a las indicaciones que García Linera ha hecho en distintos momentos durante sus múltiples conferencias.

\section{El último Lenin: pensar el Estado y su construcción}

Lo primero que habría que establecer es una periodización para hablar de el último Lenin. Nos referiremos aquí a lo que el ruso escribió o publicó entre octubre de 1920 y marzo de 1921. No daremos un tratamiento cronológico de los textos, sino más bien temático. Abordando distintos registros que contribuyen a pensar a Lenin y desde él registros usualmente fuera del "canon". Con esto nos referimos a puntos de apoyo escasamente tratados tanto por el "leninismo" de la primera coyuntura que ya explicamos, como lo referente a la segunda. Esto es así, porque como explicamos arriba, el surgimiento del "leninismo" tenía que ver con la formación del poder soviético, en tanto que el segundo momento analizado refería más bien a los distintos procesos de la revolución latinoamericana.

La intencionalidad nuestra es la de enfocar los distintos tipos de relación entre el naciente Estado revolucionario, que ha librado y superado una cruenta guerra civil con el mercado mundial. Destaca, como inicio, que señale que "El establecimiento de relaciones comerciales del país socialista con los países 
capitalistas es el factor más importante que asegura nuestra subsistencia en una situación tan compleja y absolutamente excepcional" (LENIN, 1978a, p.118). El reconocimiento de esta imperiosa necesidad, le lleva a argumentar, en distintos momentos, sobre las concesiones al capital extranjero que Rusia debe realizar. Es el caso de los bosques de Arjánguelsk, sin embargo, más allá de casos puntuales, el objetivo de Lenin era otro: "El interés por las concesiones es tan evidente que, aun cuando no lográramos otorgar una sola concesión, aún cuando ningún de nuestros acuerdos se realizada (y esto es muy posible); aún cuando así ocurriera saldríamos sin embargo ganando y deberíamos practicar esta política porque con ella dificultamos la cruzada de los países imperialistas contra nosotros" (LENIN, 1978b, p.199).

Las concesiones al capital externo tienen, entonces, un primer fin: el propiamente político. Lenin sabe que no se puede mantener una actitud beligerante de forma permanente, ni tampoco impulsar campañas de exportaciones de revoluciones. Esta no se da por la vía del cañón y el fusil, sino por medio de la acción consciente de los pueblos. Así, centra su argumento en la importancia de la construcción del Estado. Pero para ello es preciso, en primer término, parar las agresiones que vienen de las potencias y demás países capitalistas. No es, sin embargo, el único tema convocado, escribe: "nuestro interés primordial es recibir lo más rápidamente posible de los países capitalistas los medios de producción (locomotoras, máquinas, aparatos eléctricos), sin los cuales no podremos reconstruir nuestra industria más o menos seriamente..." (LENIN 1978b, p.202). Aterrizando, insiste "Para impulsar la industria química, debemos estar dispuestos a pagar mucho más a los químicos alemanes. Este es el mejor modo de aprender: entregar una concesión de las fábricas a los alemanes" (LENIN, 1978b, p.213). Como se puede notar aquí, la cuestión del comercio tiene un fin civilizatorio o industrial: la necesidad de reconstruir el conjunto de las fuerzas productivas técnicas, obtener conocimiento de su funcionamiento y poder ofrecer una continuidad de estas formas de operación de la producción. La propuesta de la concesión al capital resultó problemática, pues se dieron voces opositoras. Lenin insistió "Pero nosotros no podemos conformarnos con una victoria diplomática. Necesitamos algo más que eso, necesitamos verdaderas relaciones económicas" (LENIN, 1978e, p.25).

Los comentarios de Lenin en torno al problema de las concesiones destraba la idea de que el trayecto de la construcción socialista implica el aislamiento. Construcción soberana no es idéntico a autarquía, asilacionismo o ruptura con toda otra potencia económica. Hay necesidad de comerciar, vincularse y ampliar las relaciones con las naciones. Ello coloca en un plano estratégico la relación con el mercado mundial y la posibilidad de partir de un conjunto de fuerzas productivas técnicas mínimas o inexistentes, hacia la recuperación de ellas y su posterior potenciamiento. Lenin no apuesta a un comunismo de la escasez o del aislamiento, sino a una construcción que reconozca la necesidad del vínculo, tenso, pero crucial, con el mercado mundial. Así, teoría política y crítica de la economía anudadas en un planteamiento estratégico.

Sin embargo, no fue el único nivel en el que le dirigente ruso refirió al problema del capital. Como es bien sabido, es en esta época que se comienza a formular la Nueva Política Económica (NEP). Lo primero que habría de entender de esta acción tan importante es que fue lo que motivó su formulación.

Lenin no se tienta y escribe, sin cortapisas: "La mayoría de los campesinos sufre demasiado Germinal: Marxismo e Educação em Debate, Salvador, v. 12, n. 2, p. 86-100, out. 2020. ISSN: 2175-5604 94 
dolorosamente, el frío, el hambre y los excesivos impuestos" (LENIN, 1978, p.32). Más adelante insiste: "Reconocemos que estamos en deuda con el campesino. Hemos recibido cereales de él, a cambio de papel moneda; lo hemos tomado en préstamo, debemos devolver este préstamo y lo devolveremos mediante el restablecimiento de nuestra industria" (LENIN, 1978b, p.237).

Con esta situación económica en la que se reconoce la ruptura entre la alianza obrera y campesina se muestra que al final, no hay perspectiva trascendental, ni teleología: los intereses de una clase no son expresión universal de todas las clases explotadas. Cada clase tiene su especificidad, demanda situaciones distintas que deben ser atendidas y la coyuntura se traduce en una adecuada concesión entre las exigencias y necesidades. La labor política es la de realizar la confluencia de perspectivas diversas de las clases. Aquí, Lenin, de nuevo, no se obnubila, para lograr esto es preciso dejar crecer y fomentar las relaciones mercantiles en el campo. Primero lo coloca de esta manera: "Sería absurdo pensar que los campesinos cambiarán su actitud hacia los vitales problemas económicas como resultado de nuestra propaganda" (LENIN, 1978a, p.122). Después lo coloca como una situación no tan efímera: "Mientras tanto, durante muchos años tendremos que tratar a estos pequeños productos como a tales, y la consigna de la libertad de comercio será inevitable" (LENIN, 1978e, p.31). Queda claro el centro de la política que Lenin quiere impulsar: "Dar mayor libertad al agricultor para llevar al mercado local sus excedentes, siempre que pague sus impuestos rápida y completamente" (LENIN, 1978d, p.416). La pretensión de Lenin en este periodo es muy radical: controlar al mercado por medio del Estado.

La elaboración de Lenin no sólo deja a la teoría marxista despojada de sus reductos de filosofía de la historia (el proletariado como el representante universal y sus intereses los de todos los explotados), sino que regresa, de nuevo, al marxismo frente a la coyuntura: la necesidad de modificar la relación de fuerzas mediante la acción y decisión políticas. El trazado que hace Lenin es importante: recurrir al mercado mundial para obtener fuerzas productivas técnicas que Rusia no tiene; después reconstruir la alianza con los campesinos como forma de solventar el otro polo de la creación soberana de la industrialización. Ello es así, porque no solo hacen falta máquinas y herramientas. Para Lenin, la principal fuerza productiva son los obreros y los campesinos, quienes se encuentran en "estado de empobrecimiento, ruina, cansancio..." (LENIN, 1978e p.76), es decir, los sujetos sobre los cuales se puede construir un proyecto político estatal soberano, cuya base sea la industrialización.

Lenin asume que las concesiones al capital extranjero y en general el comercio "en todas las formas posibles, intensa y rápidamente" (LENIN, 1978h, p.187) permitirán "mejorar la situación de los obreros" (LENIN, 1978g, p.162) mediante el aumento, "a toda costa [de] la cantidad de productos. [Situación que] no podemos lograrlo sin ayuda del capital extranjero" (LENIN, 1978g, p.159). En el otro extremo, impulsa las relaciones mercantiles para que los campesinos pueden aliviar sus penurias y pobreza. El proyecto que construye debe ser entendido como el de la construcción de un Estado soberano que impulse su industria: a ello le llama capitalismo de Estado. No tiene límite al sugerir que "el capitalismo de Estado no es de temer en Rusia; sería un paso adelante" (LENIN, 1978f, p.149). Ello porque se trata de un desarrollo capitalista "orientado por el canal del capitalismo de Estado. Esto es económicamente posible, pues el capitalismo de Estado existe donde quiere que haya elementos de comercio libre y Germinal: Marxismo e Educacão em Debate, Salvador, v. 12, n. 2, p. 86-100, out. 2020. IISSN: 2175-5604 95 
capitalismo en general" (LENIN, 1978f, p.218). Lo cuál le lleva a preguntar "¿es posible combinar el Estado soviético y la dictadura del proletariado con el capitalismo de Estado? ¿Son compatibles? Por supuesto que si" (LENIN, 1978f, p.218). La ecuación de Lenin es clara: por un lado, comercio con el Occidente, para mejorar la situación obrera, por el otro, concesiones al comercio de los agricultores que permita reducir las penurias. El objetivo, construir una forma específica de desarrollo, en donde el Estado (aquí con el nombre de dictadura del proletariado) controle y encause, tanto las relaciones con el capital extranjero como con los pequeños comerciantes.

Sin embargo, existe un último registro que cierra esta triada: la transformación del Estado soviético. Aquí, nuevamente encontramos en este último Lenin una disposición conceptual y teórica distinta a la habitual, tanto aquella elaborada por los "leninistas", como por quienes intentaron realizar una lectura más profunda. En primer lugar, el reconocimiento de que el Estado soviético era una herencia del Estado zarista, es decir, que existían vínculos de continuidad; por el otro, la superación de toda rémora anarquizante a propósito de la disolución del Estado; tercero, la necesidad de su fortalecimiento, transformación y papel en la vida social.

Sobre el primer punto es fácil advertirlo en sus constantes quejas sobre la "deformación burocrática" que provenían del aparato estatal que, o bien era pre-existente o bien se organizaba espontáneamente y sin mucha claridad. En dado caso, queda claro que ni siquiera el propio Lenin consideraba que el nuevo Estado se refundaba desde cero, sino que este partía, en gran medida, de lastres del pasado, el más lacerante de todos era el de la burocracia. Así, escribe: ""Es indudable que existe una úlcera burocrática; ha sido diagnostica y es necesario tratarla seriamente" (LENIN, 1978e, p.34-35) La razón de esto la encuentra en una condición social deplorable: "Sabemos que en Rusia la rutina burocrática y el papeleo se debe principalmente al bajo nivel cultural y a las consecuencias de la extremada ruina y el empobrecimiento que produjo la guerra" (LENIN, 1978k, p.275). La burocracia es un gran problema para Lenin, quien inaugura la idea de que hay una "deformación burocrática" dentro del Estado, la gangrena no puede ser cortada de tajo.

¿Qué soluciones ensaya Lenin a este respecto? En primer lugar, Lenin actúa como suele hacerlo todo marxista, es decir, colocar sus esperanzas en que la inclusión de las masas obreras transforme de tajo esta situación: "haremos cuanto se pueda para eliminar las prácticas burocráticas promoviendo a los obreros desde abajo y aceptaremos cualquier indicación práctica en este sentido" (LENIN, 1978e, p.51). Sin embargo, esta salida choca con su diagnóstico: si una parte de la deformación burocrática se debe a la pobreza y la degradada situación social ¿cómo podrían los obreros revertir la deformación de la cual ellos mismos contribuyen? El propio Lenin tiene que ir rectificando: no hay soluciones providenciales, sino necesidad de actuar políticamente. En una carta un responsable de cierto trabajo insiste: "controlar su trabajo, llegar al fondo de los asuntos, instruirlos, enseñarles y darles una paliza. Estudiar a los hombres, descubrir a los trabajadores hábiles. Esto es ahora lo esencial..” (LENIN, 1978a p.130). Lenin comienza a rectificar a partir de la necesidad de los especialistas: "debemos tener gente apartidista que controle a los comunistas"(LENIN, 1978k, p.273). Lenin insiste, incluso con un lenguaje poco común para él, de que no basta la confirmación de una creencia política, sino la efectividad: "Aunque no estamos habituados, las Germinal: Marxismo e Educação em Debate, Salvador, v. 12, n. 2, p. 86-100, out. 2020. ISSN: 2175-5604 96 
reuniones del partido deben ocuparse de esta cuestión [del comercio] que implica espíritu emprendedor e iniciativa" (LENIN, 19781, p.320)

Lenin se queja, en este periodo, de los comunistas que quieren reinventarlo todo, de la nada: "Tenemos una cantidad terrible de entusiasta que quieren reorganizar de todas las formas posibles, y estas reorganizaciones llevan a calamidades como nunca hemos conocidos en toda mi vida" (LENIN, 1978m, p.116). Frente al comunista entusiasta, insiste en la necesidad del especialista: "Algo que no podremos lograr pronto, pero que debemos lograr a toda costa, es que los especialistas gocen de mejores condiciones de vida en el socialismo que en el capitalismo" (LENIN, 1978m, p.116). Así, asume que: "Nuestro peor enemigo interno es el burócrata, el comunista instalado en un puesto soviético responsable" (LENIN, 1978n p. 189). Todo ello es importante porque coloca la relación entre el Estado, la burocracia, las clases y los especialistas: "La idea de construir la sociedad comunista exclusivamente con las manos de los comunistas es pueril [...] Nosotros, los comunistas, no somos sino una gota de agua en el océano, en el océano del pueblo (LENIN, 1978o, p.259)

Finalmente, las reflexiones de Lenin toman un cariz distinto. Se vinculan a uno de los problemas que convocaron al mayor número de plumas a lo largo de la historia del comunismo. Por un lado, mediante la proposición del control político sobre el mercado. Este debate, que atravesará los procesos de transición y que es conocido como la vigencia o no de la ley del valor en el socialismo, reapareció con motivo de la revolución en China y de manera mucho más potente en la revolución cubana. El tema, como se sabe, es qué tanto mercado puede subsistir en el periodo de tránsito. Para Lenin es claro que el objetivo del nuevo Estado es el control de la economía mercantil: "El capitalismo de Estado es el capitalismo que debemos limitar dentro de cierto marco; pero aún no hemos aprendido a limitarlo dentro de ese marco. [...] Y de nosotros depende cómo será ese capitalismo de Estado. Tenemos suficiente poder político, del todo suficiente...” (LENIN, 1978o, p.247). Esta aseveración aparece constantemente en el último tramo de su obra. Sin embargo, remite directamente al estatuto del Estado, su determinación.

Así, sanciona que este es "un problema nuevo, pero siempre viejo" (LENIN, 1978p, p.387), es decir, el de la cualificación o determinación del Estado. Para ello Lenin proceder por dos vías. La primera convoca directamente a la transformación del Estado y su gestión por la vía de los especialistas, tema que ya se señaló antes. Para él no es posible construir con el puro voluntarismo de los militantes. Sin embargo, el segundo resulta más importante:

Estamos empeñados en la tarea de reducir el presupuesto del Estado, de reducir nuestro aparato estatal. De todas maneras, debemos reducirlo. Debemos economizar tanto cuanto sea posible. Estamos economizando en todos los aspectos, hasta en las escuelas. Debemos hacer, porque sabemos que si salvamos la industria pesada, si no la restauramos no podremos construir industria alguna...." (LENIN, 1978q, p.422)

Aquí aparece una contradicción, que ya no habrá tiempo en vida para resolver: es necesario un aparato eficiente, pero no enfermo; este aparato se aceita con la capacidad de los especialistas y de los comunistas y no ya necesariamente con la pura participación obrera; pero, además, este aparato debe ser reducido. Todo ello no sería una contradicción si Lenin no pensara en términos de un capital monopolista 
de Estado, es decir, de la necesidad de que ese aparato determinado por los distintos formatos ya mencionados, sea, además, el que regule al mercado.

\section{E1 último Lenin y América Latina}

Los procesos de transformación después de 1989 ya no aparecen como grandes revoluciones que trastornan de arriba abajo a las sociedades. Hasta ahora, en América Latina han transcurrido como acontecimientos de acceso al gobierno y de distintas escalas de transformaciones estatales, en su relación con la sociedad. El debate sobre el progresismo latinoamericano de la última década coincide en algo: la pervivencia de los aparatos estatales y sus intentos limitados -aunque importantes- de controlar segmentos del mercado.

Lenin permite acceder a una comprensión de los dilemas que enfrentó la revolución soviética. El primero de ellos es que, a pesar de la revolución, el aparato estatal se heredaba con lo que denominó "deformaciones", particularmente de tipo burocrático. El segundo fue el de la necesidad de convocar a los "expertos" para afrontar lo que los militantes y cuadros partidarios no lograban realizar con eficacia. El tercero elemento era el del burocratismo. Finalmente, todo ello era importante porque remitía a la capacidad del Estado para controlar al mercado.

Lo que tenemos es una idea de una reforma del aparato estatal que debilitara la burocracia y achicara dicho entramado institucional, pero al tiempo fortaleciera la capacidad del Estado para el control de las dinámicas de la economía mercantil. Idea contradictoria, pero central, que debe afrontarse en sus implicaciones. Un Estado fuerte es aquel que es capaz de controlar la dimensión mercantil, no es aquel que tenga un aparato más ostentoso, más amplio o más robusto. Su fortaleza depende de un objetivo, en este caso, el control y gestión de la economía, su direccionamiento. La lección dada por Lenin, no concluyente, por supuesto, es sugerente para pensar las coordenadas por las cuales atravesó en el pasado inmediato la región latinoamericana.

Lenin no ha tenido buena fama entre los teóricos que han girado en torno a estas experiencias latinoamericanos. Su vuelta, puede alumbrar segmentos importantes. Lenin coloca, en los primeros años de la experiencia soviética el andamiaje fundamental que sigue resonando en nuestros días: la construcción de un Estado, desde abajo, que permita el funcionamiento de una economía mercantil, pero que esta no salte a una dimensión plenamente capitalista. Se trata de la subordinación de la economía a las decisiones colectivas que están implicadas en la política. Lenin, como hemos seguido, apunta que es en el Estado, a partir de diversas temáticas, donde esta tensión encuentra posibilidades de resolución. Volver a Lenin, no el de los esquemas y lugares comunes, sino el de un analista de las situaciones concretas y específicas.

\section{Referencias:}

ACOSTA, V. La teoría del desarrollo capitalista en Lenin. Caracas: Universidad Central de Venezuela, 1977. 
AGUILAR, A. La teoría leninista del imperialismo. México: Nuestro Tiempo, 1978.

ALTHUSSER, L. La revolución teórica de Marx. México: Siglo XXI, 1974.

ARISMENDI, R. Lenin y la revolución en América Latina. México: Grijalbo, 1976.

BUJARIN, N. Lenin marxista. Madrid: Fontamara, 1978.

CERDA, C. El leninismo y la victoria popular. Santiago: Quimantú, 1972.

CONSUEGRA, J. Lenin y la América Latina. Colombia: Universidad del Atlántico, 1972.

DEBRAY, R. Ensayos Latinoamericanos. México: Era, 1976.

DUSSEL, E. El último Marx y la liberación latinoamericana. México: Siglo XXI, 2020.

GARCÍA LINERA, A. Condiciones de la revolución social en Bolivia (De obreros, aymaras y Lenin). La Paz: Ofensiva Roja, 1988.

HARNECKER, M. La revolución social: Lenin y América Latina. México: Siglo XXI, 1985.

LENIN, V.I. Discurso de clausura en la Conferencia de Presidentes de Comités Ejecutivos de Distritos, subdistritos rurales y aldelas de la provincia de Moscú. En: Obras completas, Tomo XXXIV. España: ECP/AKAL, 1978.

LENIN, V.I. Conferencia del PC(b)R de la provincia de Moscú. En: Obras completas, Tomo XXXIV. España: ECP/AKAL, 1978a.

LENIN, V.I. VIII Congreso de toda Rusia de Soviets. En: Obras completas, Tomo XXXIV. España: ECP/AKA, 1978b.

LENIN, V.I. La labor del comisariato del pueblo de instrucción pública. En: Obras completas, Tomo XXXIV. España: ECP/AKAL,1978c.

LENIN, V.I. Primer borrador del esbozo de tesis sobre los campesinos. En: Obras completas, Tomo XXXIV, España: ECP/AKAL, 1978d.

LENIN, V.I. X Congreso del PC(b)R. En: Obras completas, Tomo XXXV. España: ECP/AKAL, 1978e.

LENIN, V.I. Informe sobre el impuesto en especie pronunciado en una reunión de Secretarios y de representates y reponsables de la célula del PC (b) de la Ciudad y de la provincia de Moscú. En: Obras completas, Tomo XXXV. España: ECP/AKAL, $1978 f$.

LENIN, V.I. Reunión del Grupo Comunista del Consejo Central de Sindicatos de Toda Rusia. En: Obras completas, Tomo XXXV. España: ECP/AKAL, 1978g.

LENIN, V.I. A los camaradas comunistas de Azerbaidhán, Georgia, Armenia, Dagustán y de la República del Norte del Caúcaso. En: Obras completas, Tomo XXXV. España: ECP/AKAL, 1978h.

LENIN, V.I. A la conferencia de obreros apartadisitas de la ciudad de Petrogrado. En: Obras completas, Tomo XXXV. España: ECP/AKAL 1978i.

LENIN, V.I. El impuesto en especie. En: Obras completas, Tomo XXXV, España: ECP/AKAL, 1978j. LENIN, V.I. Instrucciones del CTD (Consejo de Trabajo y Defensa) a las isntituciones soviéticas locales. En: Obras completas, Tomo XXXV. España: ECP/AKAL 1978k.

LENIN, V.I. X Conferencia de Toda Rusia del PC(b)R. En: Obras completas, Tomo XXXV. España: ECP/AKAL, 19781.

LENIN, V.I. Los Sindicatos bajo la nueva política económica. En: Obras completas, Tomo XXXVI. España, ECP/AKAL, 1978m.

LENIN, V.I. Condiciones de admisión de nuevos militantes. En: Obras completas, Tomo XXXVI. España, ECP/AKAL, 1978n.

LENIN, V.I. XI Congreso del PC(b)R. En: Obras completas, Tomo XXXVI. España, ECP/AKAL, 1978 .

LENIN, V.I. Discurso de la IV sesión del CEC de todsa Rusia de la IX Legislatura. En: Obras completas, Tomo XXXVI. España, ECP/AKAL, 1978p.

LENIN, V.I. IV Congreso de la Internacional Comunista. En: Obras completas, Tomo XXXVI. España, ECP/AKAL, 1978q. LOSURDO, D. El marxismo occidental. Madrid: Trotta, 2019.

MOULIAN, T. Cuestiones de teoría política marxista: crítica de Lenin. En: Democracia y socialismo. Santiago de Chile: LOM, 2018.

MUSTO, M. Karl Marx, 1881-1883. El último viaje del moro. México: Siglo XXI, 2020.

NÚÑEZ TENORIO, J. R. Lenin y la revolución. Caracas: Editorial Revolución, 1968),

SHANIN, T. E1 Marx tardío y la vía rusa. Madrid: Revolución, 1990.

SONTAG, R. Marx y Lenin acerca de la sociología de la revolución. Caracas: UCV, 1974.

STALIN, J. Cuestiones del leninismo. En: Obras completas, tomo v. México: EDA, 1978. 
TROTSKY, L. Imágenes de Lenin. México: Era. 1970.

TROTSKY, L. Escritos filosóficos. Buenos Aires: CEIP, 2004.

ZINOVIEV, G. "El leninismo". En: El Gran debate I. La revolución permanente. México: Pasado y Presente, 1977.

\section{Notas}

1 Doutor, professor do Departamento de Política y Cultura, Universidad Autónoma Metropolitana-Xochimilco (México). Orcid: 0000-0002-8582-1216. Correo: Jaime ortega83@hotmail.com

Recebido em: 01.07.2020

Aprovado em: 25.09.2020 